Check for updates

Cite this: Mater. Adv., 2022, 3,2737

Received 8th December 2021, Accepted 3rd January 2022

DOI: 10.1039/d1ma01164b

rsc.li/materials-advances

\section{Altered phase behavior of the lauric acid-stearic acid binary mixtures in electrospun PVA-PDMS mats $\dagger$}

\begin{abstract}
Shama Perween (D) and Amit Ranjan (D) *
In this work, we study and report the phase behavior of the mixture of lauric acid (LA) and stearic acid (SA) inside polyvinyl alcohol (PVA)-polydimethylsiloxane (PDMS) nanofibrous mats woven using the electrospinning technique. Incorporating PDMS in the fibers prevents the mechanical properties from degrading due to the eutectic melting of the fatty acid mixtures present in the mats. Based on the results from scanning electron microscopy (SEM), differential scanning calorimetry (DSC), X-ray diffraction (XRD), Fouriertransform infrared (FT-IR), and Raman studies a phase diagram of the binary LA-SA mixture inside the PVA-PDMS nanofibrous mat environment has been constructed. The phase behavior suggests a eutectic composition in these mixtures of approximately 75 mole percent of LA, an intermediate composition between those obtained for the same mixture in a pure PVA nanofibrous environment ( 50 mole percent) and in the bulk ( 90 mole percent). We conjecture that the hydrophilicity/hydrophobicity of the surrounding fiber environment to which the fatty acid molecules are subjected influences the interactions between the fatty acid molecules and thereby their phase behavior. This work suggests that we can engineer the phase behavior of a fatty acid mixture by altering the properties of its nanoconfinement. From an application point of view, PDMS-PVA mats incorporating LA-SA can be used for fabricating sheets with the desired mechanical integrity and impregnated with phase change materials (PCMs) for thermal energy storage applications.
\end{abstract}

\section{Introduction}

Fibres are important one-dimensional structural and functional elements used for creating 2-dimensional and 3-dimensional objects. Electrospinning is a processing tool that produces ultrathin nanofibers enabling control of the fiber assembly properties at the molecular level. Electrospun fibers and mats have been successfully employed in various applications ranging from biomedical to energy sectors. ${ }^{1-7}$ The electrospinning process typically requires a viscous medium such as a polymer solution to form nanofibers. However, performing electrospinning using a polymer with a low glass transition temperature $\left(T_{\mathrm{g}}\right)$ is very difficult because the high mobility of the chains prevents them from remaining entangled and producing fibers. One useful polymer with an extremely low $T_{\mathrm{g}}$ is PDMS, which is non-toxic and forms an elastomer. This polymer has been extensively used in various applications, ${ }^{8-15}$ especially in the medical industry. Electrospinning has also been a frequently utilized processing technique in the medical industry. ${ }^{1}$ However,

Department of Chemical Engineering, Rajiv Gandhi Institute of Petroleum Technology, Jais, Uttar Pradesh, 229304, India. E-mail: aranjan@rgipt.ac.in $\dagger$ Electronic supplementary information (ESI) available. See DOI: 10.1039/ d1ma01164b owing to a very low $T_{\mathrm{g}}$, PDMS has evaded efforts to fabricate nanofibers via electrospinning. In earlier work, we were able to fabricate composite nanofibrous sheets that are rich in PDMS (up to around 80 weight percent (wt\%)). ${ }^{16}$ This was accomplished by blending PDMS with PVA and SA. The SA component behaved as a compatibilizer between the hydrophilic PVA and the hydrophobic PDMS components. PDMS imparts mechanical toughness to these sheets. In addition to mediating the interaction between the PVA and PDMS chains, the fatty acid (SA) component is also believed to be present in a microcrystalline form in the composite sheet. ${ }^{16}$ Since fatty acids are useful PCMs with a high enthalpy of fusion, the presence of the fatty acid micro-crystallites imparts an important functionality to these sheets: they can act as heat storage wraps to regulate the temperature of an enclosure. Thus, incorporating SA offers a route to impart multiple functionality to composite nanofibrous sheets: it (i) facilitates electrospinning of the PDMS, which improves the mechanical behavior of the sheets; (ii) allows the sheet to be used as heat storage wrap; and (iii) helps in controlling the hydrophilicity and hydrophobicity so as to facilitate the formation of selectively permeable membranes. ${ }^{16}$

The well-established eutectic phase behavior of the fatty acid mixtures ${ }^{17-19}$ has been an important driving force towards their use as PCMs in thermal energy storage applications. An 
important drawback with these eutectic mixtures is that upon melting they tend to spill out of the medium, thereby restricting its utility. One possible solution as reported in previous work is to incorporate them in PVA mats. ${ }^{20}$ It was observed that the problem was only partially solved since the mechanical properties of the mat serving as the medium worsened upon eutectic melting. In order to overcome this limitation, we introduce a third component to PDMS to improve the mechanical integrity of the mats. In this paper, we perform a detailed study of composite nanofibrous sheets of PDMS, PVA, and fatty acid binary mixtures, by fixing the PDMS and PVA components but varying the composition of the fatty acid component from pure LA to pure SA. The morphological, chemical, structural, and thermal behavior of the fibers has been characterized using SEM, FT-IR, XRD and DSC and correlated with the composition of the fatty acid mixture. Mixtures confined to nanoscopic dimensions may show altered phase behavior compared with the bulk. A phase diagram of the LA-SA mixture has been constructed in this work. In earlier work, ${ }^{20}$ the phase diagram of these binary mixtures of fatty acids when present in the PVA nanofibrous sheets showed a significant drop in eutectic temperature compared with the bulk mixtures, and also a shift in eutectic composition. In this work, we find that incorporating PDMS in the sheets as the majority component along with PVA does not affect the eutectic temperature of the mixture significantly compared with that in the sheets that have fatty acid mixtures with PVA alone. However, a significant shift in the eutectic composition is observed. The eutectic composition of the LA-SA mixtures in the PDMS-PVA composite sheet observed in this work $\left(X_{\mathrm{LA}} \approx 0.75\right)$ is intermediate between the values observed in the PVA nanofibrous sheets $^{20}\left(X_{\mathrm{LA}}=0.58\right)$ and the bulk $\left(X_{\mathrm{LA}}=0.85\right) .{ }^{17}$ The change in eutectic point may not be surprising, since the interactions may be altered due to medium confinement. However, to the best of our knowledge, such observations have not yet been reported. Secondly, we aim to emphasize the trend in the eutectic composition, which can be changed by changing the hydrophilicity $v s$. hydrophobicity of the fibrous medium. The main aim of this paper was to establish and characterize this aspect, which bears importance in their potential technological applications such as in sheets for heat storage.

In addition to showing a significantly altered phase behaviour, the mats also showed much improved mechanical behaviour compared with the case when only PVA is used and the PDMS component is absent. However, the analysis of mechanical behaviour will be presented elsewhere. In this paper, we focus on uncovering the physicochemical aspects that result in the altered phase behaviour in these systems.

\section{Experimental section}

\subsection{Raw materials}

Sylgard-184 from Dow Corning, Midland, MI, USA is used as working PDMS elastomeric material. Polyvinyl alcohol (PVA) of $M_{\mathrm{w}}$ ranging from 85000 to 124000 (used as a guiding polymer in electrospinning) and powders of lauric acid (LA) and stearic acid (SA), which were used as the composite PCM, were purchased from SD Fine Chem. Ltd (Bombay, India); these were used without further purification. Tetrahydrofuran (THF, 99.5\%) was purchased from SD Fine Chem. Ltd and was used as a solvent to swell the cross-linked PDMS. Absolute methanol (99.0\%) was purchased from SDFCL SD Fine Chem. Ltd. DIwater was used to make all PVA solutions and emulsions.

\subsection{Preparation of spinning mixture solutions}

Three stock solutions, those of PVA in DI water, the LA-SA mixture in methanol, and PDMS in THF, were prepared separately. Firstly, a 10\% PVA solution in DI water was prepared via mixing and stirring for $3 \mathrm{~h}$ at $60{ }^{\circ} \mathrm{C}$ before it was left to cool till it reached room temperature (RT). Then the second solution of SA and LA in methanol ( $4 \%$ by weight) was prepared at RT. The third solution of PDMS (40\% by weight) without cross-linker was prepared in THF at RT. The binary mixture solution of LA-SA with varying mole fractions was added dropwise into the aqueous PVA followed by the addition of PDMS solution. The PVA and PDMS composition in the final spinning solution was kept constant while the binary fatty acid mixture composition was altered from 0.1 to 0.9 molar ratio of SA. Overall, nine final mixtures were prepared for electrospinning, whose details are tabulated in Table 1, each of which has a fixed PDMS weight percentage of $74.07 \%$ and a PVA weight percentage of $18.52 \%$. The spinning solution has an overall solute weight fraction of $\sim 18 \%$, consisting of PDMS, PVA, and fatty acids. The fixed weight of the solute has a fixed PDMS weight percentage of $74.07 \%$ and a PVA weight percentage of $18.52 \%$ with the remaining being fatty acids in all cases. It was the fatty acid composition (LA to SA fraction) that was varied in each mixture.

\subsection{Electrospinning (emulsion electrospinning technique)}

Nanofiber mats were fabricated using a mixed solution of the binary mixture of LA-SA, PVA, and PDMS in the electrospinning setup, which was purchased from E-Spin Nanotech Pvt. Ltd, India. A $10 \mathrm{~mL}$ plastic syringe with a stainless-steel needle at the tip was filled with the final spinning solution. During electrospinning, a positive high voltage of $15 \mathrm{kV}$ was applied to the needle, and the fibers were collected on an aluminum foil wrapped around an electrically grounded rotating collector drum as the cathode. The needle-to-collector distance was kept in the range of $10-12 \mathrm{~cm}$ and the solution flow rate was maintained using a syringe pump. The temperature and the

Table 1 Nomenclature of samples and the mixture composition of LA-SA with PVA-PDMS

\begin{tabular}{lll}
\hline Sample name & $\begin{array}{l}\text { Weight-fraction of LA } \\
\text { in LA-SA mixture }\end{array}$ & $\begin{array}{l}\text { Mole fraction of LA in } \\
\text { LA-SA mixture }\left(X_{\mathrm{LA}}\right)\end{array}$ \\
\hline LA-SA-1 & 1.000 & 1 \\
LA-SA-2 & 0.875 & 0.91 \\
LA-SA-3 & 0.750 & 0.81 \\
LA-SA-4 & 0.625 & 0.70 \\
LA-SA-5 & 0.500 & 0.59 \\
LA-SA-6 & 0.375 & 0.46 \\
LA-SA-7 & 0.250 & 0.32 \\
LA-SA-8 & 0.125 & 0.17 \\
LA-SA-9 & 0.00 & 0.00
\end{tabular}


relative humidity of the spinning chamber were, respectively, $26 \pm 0.1{ }^{\circ} \mathrm{C}$ and $40 \pm 1 \%$. The method and the parameters used for the electrospinning technique for fibrous mat preparation are provided in the ESI, $\dagger$ Section S.1.4.

The details of all methods and techniques are provided in the ESI, $\dagger$ Section S.1.

\section{Results and discussion}

\subsection{Morphology and elemental mapping}

The figures represent the FE-SEM images for all the binary mixture compositions from a higher to lower mole fraction of LA. Fig. 1(a and j) represents the images at lower magnification and Fig. 1(b-i) present the images of the electrospun mat with decreasing LA composition in the mat at high magnification. Beading is found to monotonically decrease with increasing SA composition in the fixed LA-SA mixture fraction. Considering the facts that the composition of the fatty acid mixture is only about 7 percent in all the samples and the viscosity values of the neat fatty acids are at least an order of magnitude smaller than that of the spinning solution, the viscosity values in different spinning solutions should not differ appreciably so as to influence the beading behavior. The zero shear viscosity values of the spinning solutions containing pure LA and pure SA are nearly equal (0.1 Pa s, please see the ESI, $\dagger$ Fig. S1). Therefore, it is quite remarkable to observe an improvement in the beading behavior with a change in the fatty acid composition. A theoretical explanation could be offered based on the model of the molecular arrangement proposed in ref. 16. When fatty acid molecules bind to the PVA backbone and extend out laterally, they form a grafted polymer with a comb-like architecture (also referred to as a bottlebrush polymer). It has been shown that the crowding parameter $(\Phi)$, defined as the ratio of the total monomer volume of a polymer to the pervaded volume of the polymer, has the following dependency: $\Phi \approx \varphi^{-1} n_{\mathrm{SC}}{ }^{-2}$ where $\varphi$ is the volume fraction of the backbone monomers in the graft macromolecules, and $n_{\mathrm{SC}}$ is the number of monomers in the side chains grafted to the main backbone. ${ }^{21,22}$ In our samples, $\varphi$ is the same in each case, but replacing LA with SA increases $n_{\text {SC }}$ such that $\Phi$ decreases. As a result, in the pervaded volume of a given PVA chain, there is a larger number of other chains. As a given chain interacts with a larger number of other chains, it leads to better fiber formation and hence less beading.
The EDS spectra (Fig. 2) in the samples with pure LA give a very small weight percentage of silicon in a spot chosen on the bead $(1.12 \%)$ but give a relatively large weight percentage of silicon over an extended area (5.56\%). This is not observed in SA-rich samples. From the difference between the measured weight percentage of silicon in the bead and over the extended area in the pure LA mats, we can conclude that the PDMS chains have been partitioned, and also that SA helps in dispersing the PDMS molecules much better than LA. The difference in the weight percentage values of silicon over the extended area measured via EDS and from the known composition of the mat can be attributed to the fact that the electron beam is being screened by the beads and hence the composition of silicon measured via EDS may not be a true representation of the actual value. These results suggest that the beading can be attributed to the fatty acid components. We aim to minimize the beading since it adversely affects the mechanical strength of the fiber. However, beads incorporated on string fibers in the micron range have potential applications in tissue engineering, drug delivery, as well as in air and water filters. ${ }^{23-29}$ Fig. 3 shows that the formation of the beads, quantified by the area fraction of the beaded region in the images, is greater in the case of pure LA compared with pure SA. It is also to be noted that in the samples containing LA-SA mixtures with $X_{\mathrm{LA}}=0.81$, no fibers are observed. This is consistent with other characterization results, which suggest that this composition is close to the eutectic composition. At this composition, the mixture melts easily and thereby, owing to its fluidity, prevents the fiber formation.

\subsection{Atomic force microscopy}

The PVA-PDMS nanofibrous films formed after being combined with the fatty acids show excellent selective permeability to oil compared with water. ${ }^{16}$ However, surface permeation is dependent on the contact angle, which in turn depends on the surface roughness. Here we utilize AFM to characterize the surface topography and roughness. Fig. 4 shows the AFM image and the surface topography of the mats with four different LA fractions, and the topographical data for the respective images in Fig. 4 are presented in Table 2 . We characterize the roughness by relating it to the ratio of the amplitude to the wavelength (in-plane correlation length) of any undulation, which is obtained from the line-scans of the image. The AFM line scans are visually inspected and the mean values of this ratio for
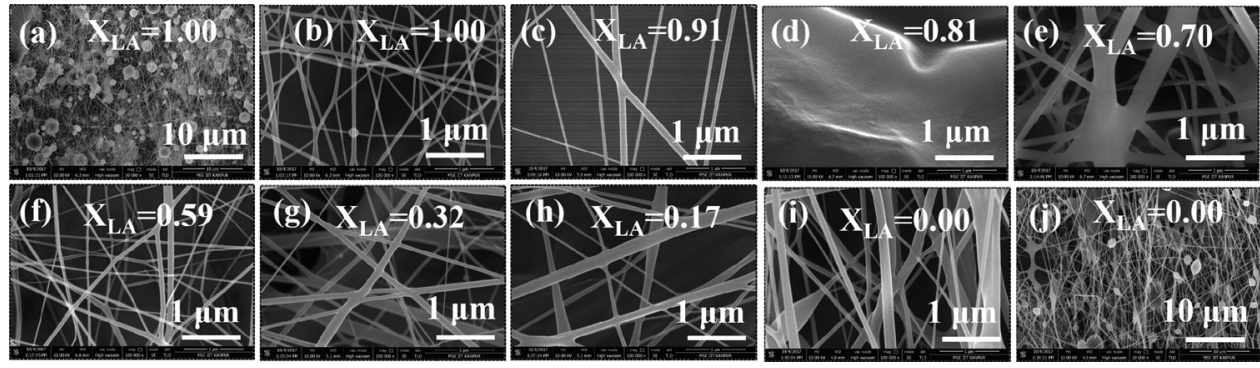

Fig. 1 FESEM images of the LA-SA-PVA-PDMS mat: (a) and (j) pure LA $\left(X_{L A}=1\right)$ and pure SA $\left(X_{L A}=0\right)$, respectively, in the PVA-PDMS mat at the scale bar of $10 \mu \mathrm{m}$; and $(\mathrm{b}-\mathrm{i})$ binary mixture of LA-SA with PVA-PDMS with decreasing composition of LA at higher magnification (scale bar $=1 \mu \mathrm{m})$. 

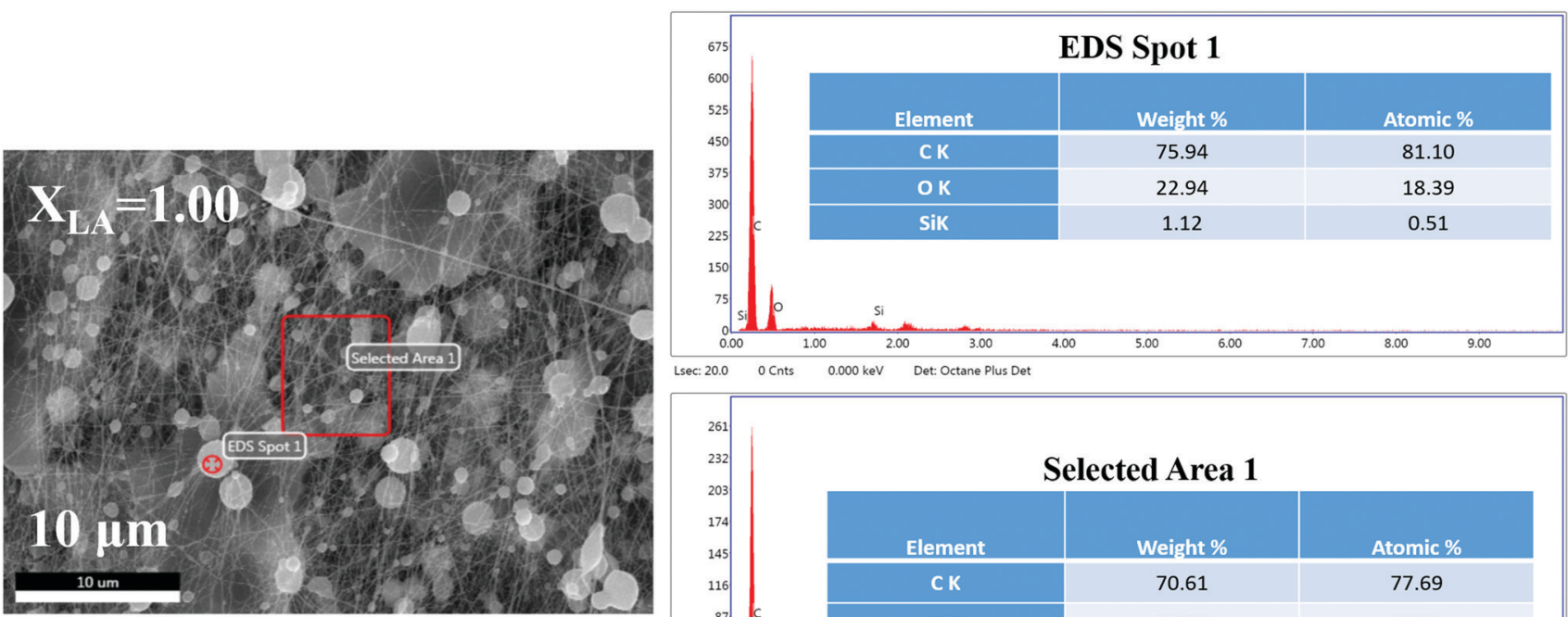

Lsec: $20.0 \quad 0$ Cnts $0.000 \mathrm{keV} \quad$ Det: Octane Plus Det

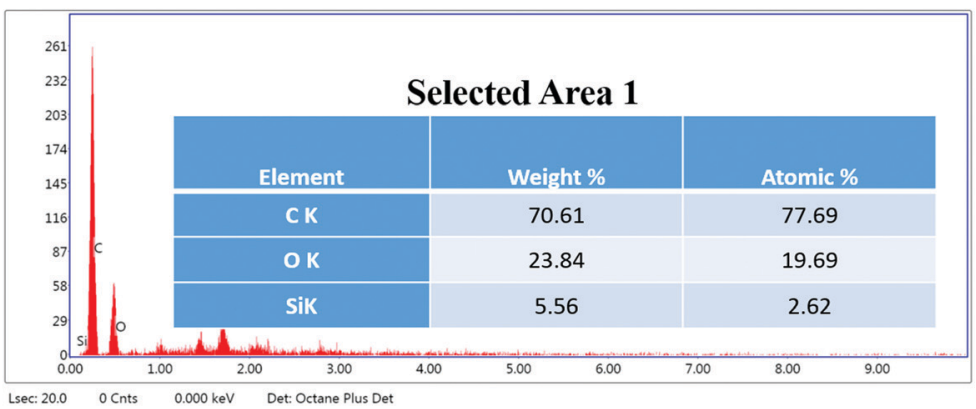

Fig. 2 Energy dispersive spectra of the electrospun mat $\left(X_{\mathrm{LA}}=1.00\right)$ showing the elemental distribution.

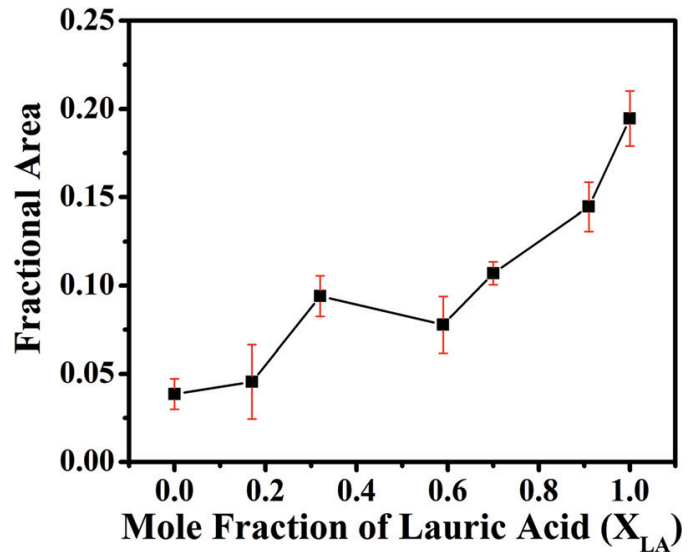

Fig. 3 Fractional area of beads present in nanocomposite fibrous mat images. Data were obtained by analysing the SEM images at a magnification of $25000 x$

different samples are estimated from the statistics of multiple data. The trend in the surface roughness presented in Fig. 5 suggests that the incorporation of SA improves the roughness, which can be attributed to the higher bead-forming tendency with LA.

The Table 2 lists the values of the height variation, the corresponding correlation length, and their ratios in respective columns as presented in Fig. 4(a-d). These values are obtained from visual inspection.

\subsection{Phase studies using X-ray diffraction}

The fatty acid molecules assume various crystalline arrangements called polymorphs. ${ }^{30}$ These arrangements differ in terms of their unit cell symmetry and geometry. Among these polymorphs, some are stable while others may be metastable at RT. The observed polymorphs are strongly dependent on the processing conditions. For example, the reported polymorphs of LA are C (metastable), $A_{1}, A_{\text {super }}$ whereas the SA polymorphs include $A_{1}, A_{\text {super }}, B_{o}, B_{m}, C$, $\mathrm{E}_{\mathrm{o}}$, and $\mathrm{E}_{\mathrm{m}} \cdot{ }^{31}$ Owing to a longer carbon chain, the crystalline phase of SA has a larger scope of polymorphic alternatives compared with LA. Crystallinity in fatty acids has been well studied. The major XRD reflections due to LA and SA arise at $2 \theta$ values of around $10^{\circ}$ or less, with some higher-order reflections appearing at higher $2 \theta$ values. PVA is another component that may give an XRD signal due to its semi-crystalline nature. The primary XRD reflection due to PVA appears at $2 \theta=20^{\circ}$. Here we do not investigate the details of the XRD reflections but only utilize these characteristic XRD patterns of PVA and fatty acid in analysing the trends in our data. For the sake of brevity, the detailed XRD reflection assignments based on the previous literature $^{30-52}$ are listed in the ESI $\dagger$ (Table S1). The XRD patterns of the mats with varying compositions along with the reference diffraction patterns (Crystallographic Information File (.cif) database from Cambridge Structural Database (CDS) for PVA, LA and SA) are presented in Fig. 6. It can be seen from these figures that as SA is added to LA, the crystallinity of the fatty acids is initially suppressed, but in samples with the SA fraction above $0.3\left(X_{\mathrm{IA}}<0.7\right)$ the crystallinity reappears, as suggested by the trend in the sharp reflections with $2 \theta$ values of less than $10^{\circ}$. These XRD patterns clearly support the conclusion that the crystallinity of the fatty acid molecules is suppressed in the composition range $0.9<X_{\mathrm{LA}}<0.7$, as corroborated by other data presented later in the paper, and suggest a eutectic composition in this range.

\subsection{ATR-FTIR/Raman spectroscopy}

The entire FT-IR spectra for different mats are presented in Fig. S2 in the ESI, $\dagger$ and only important bands are presented and analysed here. We first analyse the IR bands that are chiefly 

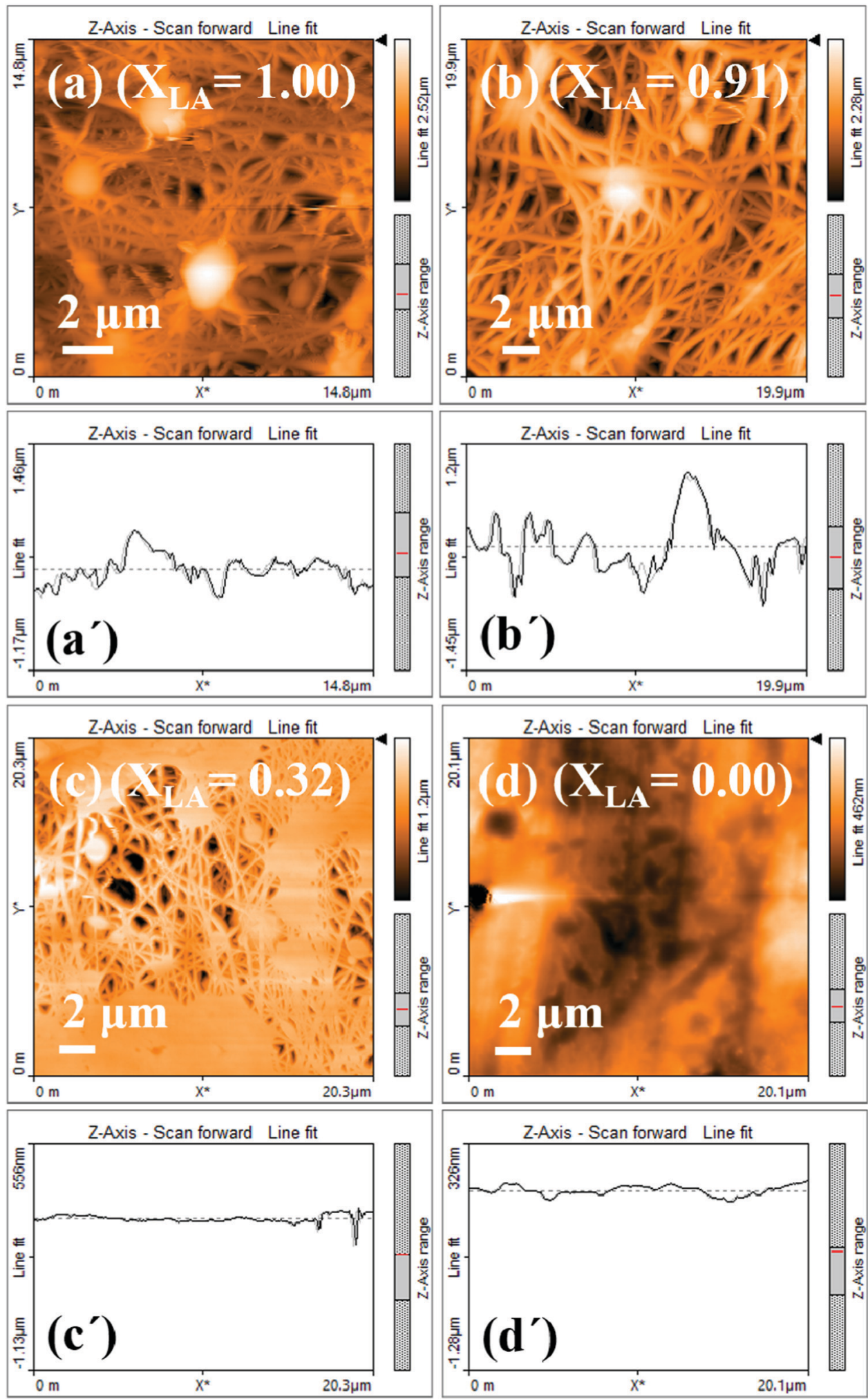

Fig. 4 AFM images of (a) $X_{\mathrm{LA}}=1.00$, (b) $X_{\mathrm{LA}}=0.91$, (c) $X_{\mathrm{LA}}=0.32$ and (d) $X_{\mathrm{LA}}=0.00$ present the surface topography, with the length of line scans being $14.8 \mu \mathrm{m}, 19.9 \mu \mathrm{m}, 20.3 \mu \mathrm{m}$ and $20.1 \mu \mathrm{m}$, respectively. The top right color bar represents the relative vertical profile. $\left(a^{\prime}\right)$, ( $\left(b^{\prime}\right)$, $\left(c^{\prime}\right)$ and $\left(d^{\prime}\right)$ Corresponding average height variation of (a), (b), (c), and (d), respectively. The surface depth height: (a) 0 to $2.52 \mu \mathrm{m}$, (b) 0 to $2.28 \mu \mathrm{m}$ and (c) 0 to $1.2 \mu \mathrm{m}$.

attributable to the PDMS compound, since it is the majority component present in our mats. Stretching of the Si-O-alkyl group in the PDMS chains gives a strong band in the range
1000-1100 $\mathrm{cm}^{-1}$ with two separated peaks near 1020 and $1090 \mathrm{~cm}^{-1}$ as shown in Fig. 7(a). This band has been observed and studied in detail in silica and other materials that consist 
Table 2 Line scan parameters measured for the four cases presented in Fig. 4

\begin{tabular}{|c|c|c|c|c|c|c|c|c|c|c|}
\hline & \multicolumn{5}{|c|}{$X_{\mathrm{LA}}=1$} & \multicolumn{5}{|c|}{$X_{\mathrm{LA}}=0.91$} \\
\hline Correlation length, $W(\mathrm{~nm})$ & 1298 & 695.8 & 1102 & 927.9 & 811.8 & 2879 & 1634 & 2490 & 2880 & 1712 \\
\hline$D / W$ & 0.402 & 0.32 & 0.733 & 0.865 & 0.679 & 0.29 & 0.246 & 0.275 & 0.263 & 0.262 \\
\hline & \multicolumn{5}{|c|}{$X_{\mathrm{LA}}=0.32$} & \multicolumn{5}{|c|}{$X_{\mathrm{LA}}=0$} \\
\hline Depth, $D(\mathrm{~nm})$ & 266.5 & 315.5 & 288.1 & 188 & 74.56 & 37.5 & 46.87 & 75 & 120 & 56.25 \\
\hline
\end{tabular}

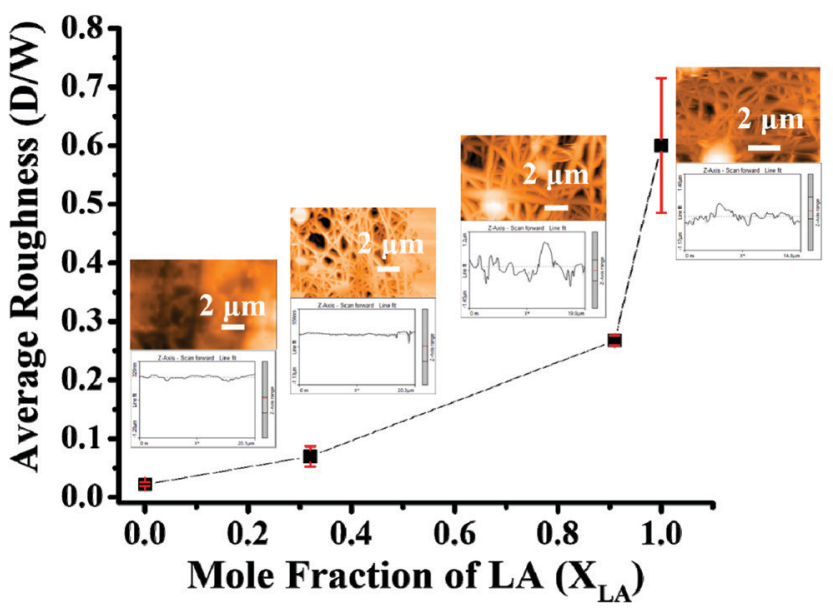

Fig. 5 Average surface roughness of the mat as a function of the mole fraction of LA. The images and the line scans presented next to the data points are the same as in Fig. 4.

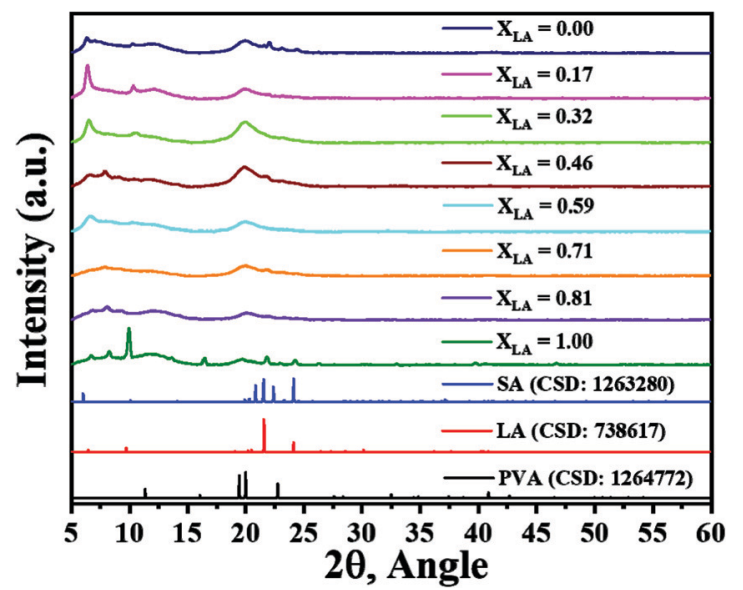

Fig. 6 X-ray diffraction patterns of electrospun mats with a decreasing LA mole fraction in the PVA-PDMS compositions from bottom to top. The bottommost three diffraction patterns belong to the reference compounds PVA, LA and SA, as labelled.

of $\mathrm{SiO}_{4}$ tetrahedral units. ${ }^{53-55}$ The two peaks are assigned respectively to four-membered and six-membered rings of the tetrahedra with the higher energy peak corresponding to the six-membered rings, which have a larger $\mathrm{Si}-\mathrm{O}-\mathrm{Si}$ bond angle. ${ }^{56,57}$ In our mats we also attribute these two peaks to two different conformations of the PDMS backbone, which may assume different possible bond angles of the $\mathrm{Si}-\mathrm{O}-\mathrm{Si}$ bonds. These two peaks show a non-monotonic redshift as the LA:SA composition in the mats is varied, with the maximum redshift occurring for the intermediate compositions of LA lying in the range of $0.7-0.9$. This suggests that in this composition range, the $\mathrm{Si}-\mathrm{O}-\mathrm{Si}$ bond angle tends to decrease compared with other compositions. The maximum shift is shown by the red curve in Fig. 7(a), which corresponds to the LA: SA composition of 0.9. As suggested by other characterization data presented later, the LA-SA mixture also shows a eutectic composition in this range. Hence, we attribute this shortening of the $\mathrm{Si}-\mathrm{O}-\mathrm{Si}$ bond angle to better intermixing of the fatty acid components, which in turn mediates the interaction between the PVA and PDMS chains via hydrophobic interactions between the alkyl group attached to Si in PDMS and the carbon backbone of the fatty acids. In addition, the intensity of this band also increases in these samples. These observations imply that PDMS is better dispersed in samples with the LA:SA ratio close to 0.9 due to better intermixing of LA and SA, which helps in compatibilizing the hydrophilic PVA and hydrophobic PDMS molecules. ${ }^{16}$ Another prominent band due to PDMS appears near $1260 \mathrm{~cm}^{-1}$ due to the $\mathrm{CH}_{3}$ umbrella deformation from Si-CH . As seen in Fig. 7(c), for the aforementioned intermediate LA:SA compositions a prominent band is observed at $1260 \mathrm{~cm}^{-1}$. However, as the composition is varied away from this range, this band becomes broader and a small shoulder appears in the signal. This suggests that at LA:SA compositions away from the range that presumably enhances the intermixing, the PDMS molecules experience inhomogeneous surroundings. The bulk mixture of LA-SA shows a eutectic phase behavior, wherein molecules exhibit better intermixing at the eutectic composition (and temperature) and partition into crystalline phases at compositions away from the eutectic compositions. $^{20,58}$ Hence, the proposed inhomogeneity in our mats that leads to a broadening of the $1260 \mathrm{~cm}^{-1}$ band could arise due to the presence of microcrystallites of LA and SA molecules, which may segregate at such off-eutectic compositions. This conclusion is also supported by our DSC and XRD data as discussed later. However, as presented later, the LA-SA eutectic composition significantly deviates in our mats compared with their value in the bulk, which can be attributed to the altered interaction between the fatty acid components induced by an 


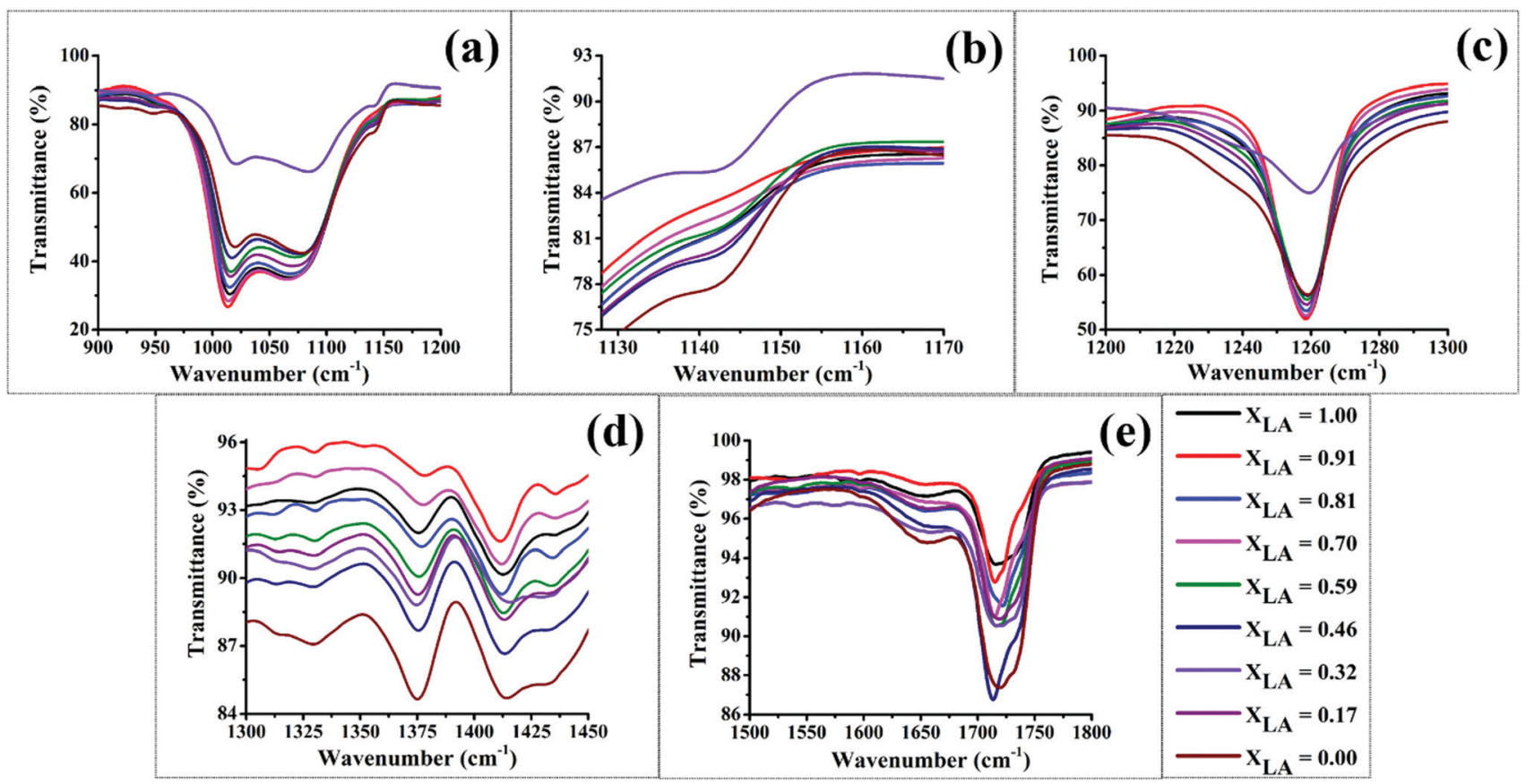

Fig. 7 FT-IR spectra of as electrospun deposited mats for all the LA-SA mixtures in PVA-PDMS. (a) to (e) correspond to different range of wavenumbers.

altered nanoconfinement in terms of the hydrophilicity and hydrophobicity.

PVA gives a weak signal in the infra-red spectrum at a wavenumber close to $1141 \mathrm{~cm}^{-1}$. This absorption is attributed to the crystallinity of PVA, as seen in Fig. 7(b). The IR signal due to crystallinity in PVA weakens for LA:SA compositions in the range of $0.7-0.9$ but is recovered for other compositions. This observation suggests that the PVA crystallinity is suppressed in these samples, which further lends support to the aforementioned conclusion of optimal intermixing of the two fatty acid components occurring in this range of composition, which helps the fatty acids to better spread in the medium and disrupt the ordered arrangement of PVA sheets. Another notable trend can be observed in the peak appearing at $1374 \mathrm{~cm}^{-1}$, which has been attributed to the $\mathrm{CH}_{3}$ umbrella deformation in $\mathrm{C}-\mathrm{CH}_{3}$. Fig. 7(d) shows that the relative intensity of this IR peak reduces in the mats with the LA:SA composition in the range of 0.7 to 0.9 . This suggests that the terminal $\mathrm{CH}_{3}$ group in the fatty acids become increasingly hindered due to the increased hydrophobic interaction with the PDMS in this composition range. This observation is also in agreement with the conjecture that LA and SA molecules undergo better intermixing for this composition range, which helps them spread more uniformly and connect the PVA and PDMS in a better fashion. At other compositions, the fatty acid components prefer to segregate to form crystalline microdomains. This conclusion is also supported by our XRD data presented earlier (see Fig. 6). Near the eutectic compositions, the fatty acid molecules of either kind tend to form dimers, which result in weakening of the $\mathrm{CO}$ stretching vibrations. The $\mathrm{C}=\mathrm{O}$ stretching frequency of a carboxylic group is an excellent vibrational spectral marker for detecting the protonation state of this group. ${ }^{59-64}$ The weakening of the $\mathrm{CO}$ bond is reflected in the non-monotonic redshifts in the IR band near $1700 \mathrm{~cm}^{-1}$, as shown in Fig. 7(e). The maximum redshift is observed for $X_{\mathrm{LA}}=0.81$.

The proposed fluidity of the fatty acid molecules is also corroborated by examining the Raman spectrum in the Raman shift range of 1000-1140 $\mathrm{cm}^{-1}$. In the Raman study of aliphatic carbon chains (present in fatty acids), the peaks appearing at the Raman shifts of 1056 and $1100 \mathrm{~cm}^{-1}$ have been attributed to solid crystalline ordering resulting from all-trans conformations, but an intermediate peak at $1075 \mathrm{~cm}^{-1}$ is attributed to several gauche conformations present in the fluid-like aggregation. The relative intensity of these sets of peaks is a good measure of fluidity $v s$. crystallinity in fatty acid molecules. ${ }^{65,66}$ From the Raman spectra of the four samples presented in Fig. 8, we observe that in the sample with pure LA, the peak owing to crystalline fatty acid is more intense compared with that from the fatty acid in a fluid state. But as the SA is introduced, the peaks become nearly equal in strength, thereby suggesting the prevalence of the fluid-like state of the fatty acid molecules.

The IR data complemented by the Raman data suggest the following overall picture. The fatty acid molecules have two roles: mediating the interaction between the PVA and PDMS and forming their own microcrystalline domains. The tendency of the fatty acids to form their own microcrystalline domains is suppressed near the eutectic composition and two different fatty acids become better compatible, spread out in the medium in the form of dimers, ${ }^{61,62}$ and in the process of compatibilizing the PVA and PDMS molecules, they suppress the crystallinity of the PVA molecules.

\subsection{Phase behavior using differential scanning calorimetry}

In this section, we study the phase behavior of the fatty acid binary mixtures where other components of PVA and PDMS present in the form of nanofibers are treated as an immersing 


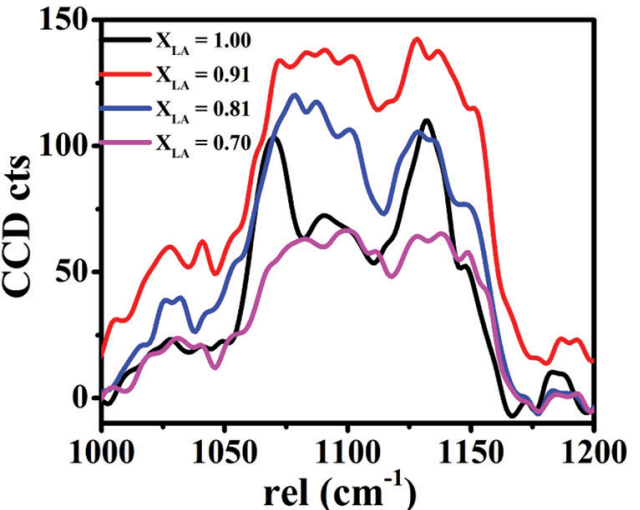

Fig. 8 Raman spectra of electrospun mats.

medium. The medium present in the form of nanofibers causes these molecules to become confined over a length scale of a few hundred nanometers.
DSC traces of the second heating of all the compositions of LA-SA mixtures with PVA-PDMS are presented in Fig. 9. The possible transitions in the materials are the melting of LA, and SA, multiple polymorphic solid-solid transformations in LA and $\mathrm{SA}$, and the glass transition in PVA. Moreno et al. have performed a detailed study of polymorphs and possible transitions in even saturated fatty acids and reported a solid-solid transition. ${ }^{30}$ LA exists in the $A_{1}, A_{\text {super }}$ and $C$ forms whereas $S A$ is known to exist in the $\mathrm{A}, \mathrm{B}, \mathrm{C}$ and $\mathrm{E}$ forms with $\mathrm{B}_{\mathrm{o} / \mathrm{m}}$ and $\mathrm{E}_{\mathrm{o} / \mathrm{m}}$ polytypes, where ' $\mathrm{O}$ ' and ' $\mathrm{m}$ ' represent the orthorhombic and monoclinic cells, respectively. ${ }^{30,31,33,42,47,49,52}$

The thermal transitions in LA and SA have been studied in detail in the past. Sato and Boistelle ${ }^{67}$ have studied the thermodynamic stability of these polymorphs, where they reported that the B and C forms of SA are stable below and above $32{ }^{\circ} \mathrm{C}$, respectively, while the $\mathrm{A}$ and $\mathrm{E}$ forms are considered as metastable phases within the whole temperature region. The transition in SA from $\mathrm{B}$ to $\mathrm{C}$ has also been observed at $46{ }^{\circ} \mathrm{C}^{50}$
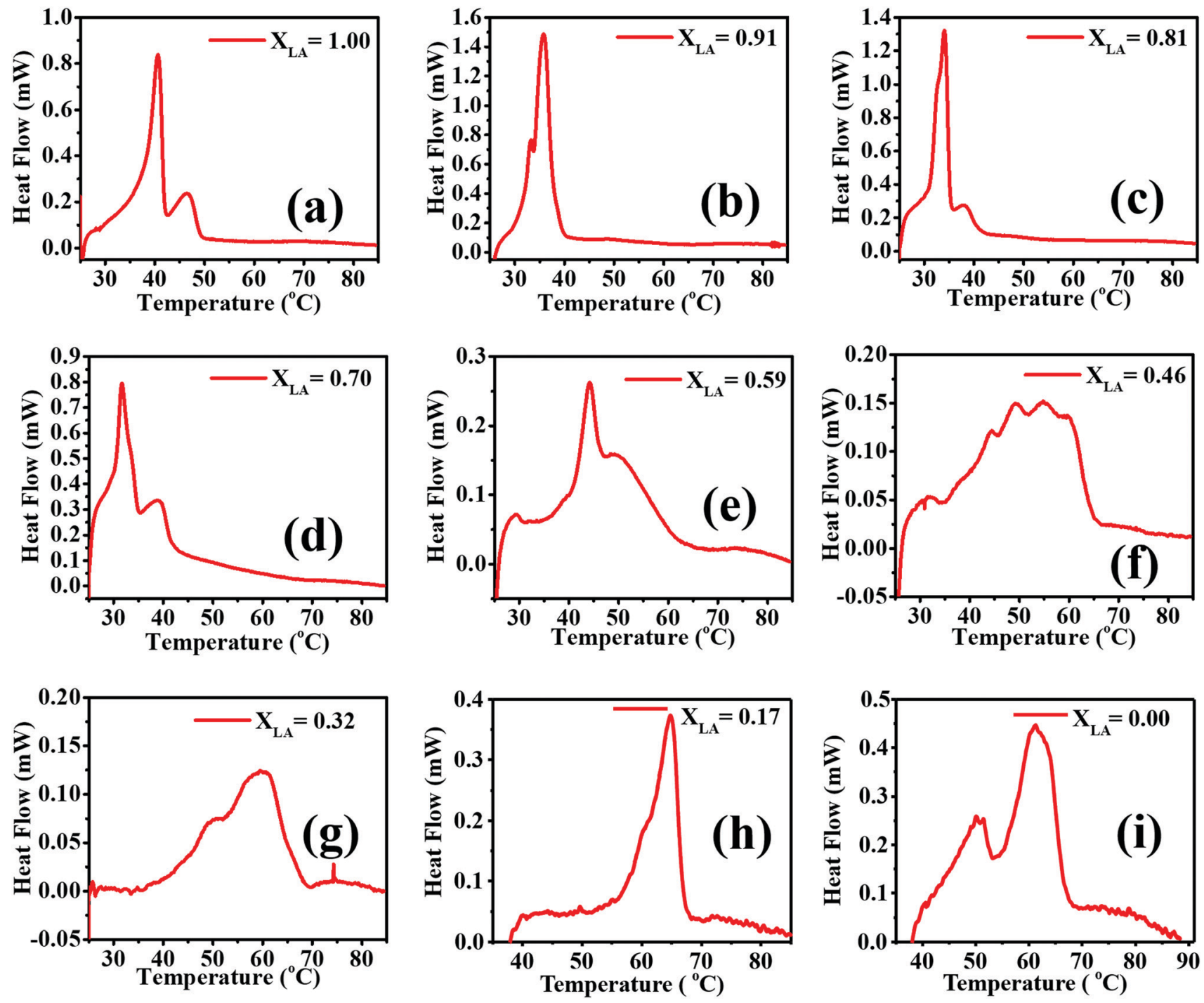

Fig. 9 DSC second heating scan of the electrospun mats for all the LA-SA mixture compositions. (a) to (i) correspond to different values of LA mole fractions. 
and $54{ }^{\circ} \mathrm{C}^{48,66}$ The scatter in the transition temperature data reported by various researchers can be attributed to the presence of a kinetic barrier that may be influenced by the processing parameters such as the heating rate, solvents, and the presence of other components. The $\mathrm{B}$ and $\mathrm{C}$ forms in $\mathrm{SA}$ co-exist at $32{ }^{\circ} \mathrm{C},{ }^{30,31,34,49,67-69}$ whereas in LA the solid-solid transitions from $\mathrm{A}_{\text {super }}$ to $\mathrm{C}$ and $\mathrm{B}$ to $\mathrm{C}$ occur at $35.1{ }^{\circ} \mathrm{C}$ and $10.1{ }^{\circ} \mathrm{C}$, respectively. ${ }^{30,46}$

We have performed analysis of the DSC thermograms obtained for our samples based on the reported transitions in the literature cited above (see ESI, $\dagger$ Table S2). We have performed DSC analysis of both the as-spun (during the first heating) and annealed (during the second heating) samples. For the sake of brevity, the first heating scans are presented in ESI, $\dagger$ Fig. S3, and here we only present the second heating scans. In almost all the as-spun samples in the first heating scans, including those with pure LA, a weak and broad peak is observed at a temperature close to $48{ }^{\circ} \mathrm{C}$, in addition to a melting peak of LA close to $42{ }^{\circ} \mathrm{C}$. This peak does not appear in the DSC thermograms of the same samples in the second heating. In the electrospun mats with pure LA and PVA, a similar peak has been observed (Fig. S4, ESI $\dagger$ ). Therefore, we ascribe this peak near $48{ }^{\circ} \mathrm{C}$ to a polymorph of LA, the formation of which is promoted due to nanoconfinement by the PVA nanofibers. Multiple melting events due to the nanoconfinement of LA have also been observed by Mitran et al. ${ }^{70}$ The transitions of the electrospun mats with a pure LA composition are observed at around $32{ }^{\circ} \mathrm{C}$, which is due to the melting of the LA crystals, and in the case of pure SA two transitions at around $53{ }^{\circ} \mathrm{C}$ and $64{ }^{\circ} \mathrm{C}$ are observed. The first transition has been attributed to the $\mathrm{B}$ to $\mathrm{C}$ transformation, as also reported by Singleton et $a .^{71}$ Interpreting the various thermal transitions observed in the thermograms as polymorphic transitions reported in the literature, we have constructed a binary phase diagram of LA and SA treating the majority components of PDMS and PVA as constituting the medium that accommodates the fatty acid molecules. The phase diagram based on the second heating scan is presented in Fig. 10 (ESI, $\dagger$ Table S3). We can arrive at the following conclusions from the phase diagram: (a) The formation of different polymorphs is promoted in pure SA due to the confinement imposed by the nanofibrous medium. Stearic acid is known to have several metastable phases, the formation of which may be kinetically favoured due to confinement. However, at this stage we are unable to characterize the structures of the polymorphs obtained after undergoing various thermal transitions. Therefore, we are, at this stage, unable to provide a picture of how the molecular aggregation pattern changes upon the thermal transitions. (b) These polymorphs persist even upon the addition of LA and coexist with a homogeneous mixture in the molten liquid phase. (c) For a LA fraction less than $\sim 30$ mole percent, SA can accommodate LA molecules to form a homogeneous crystalline phase (referred to in Fig. 10 as $\alpha$ ). (d) A eutectic temperature can be identified at a temperature close to $32{ }^{\circ} \mathrm{C}$ at a eutectic composition of 0.75 .

In an earlier study, the LA-SA mixture was spun with PVA and a similar study on its phase behavior was carried out. ${ }^{20}$ With the PVA nanofibrous medium, the binary mixture of LA

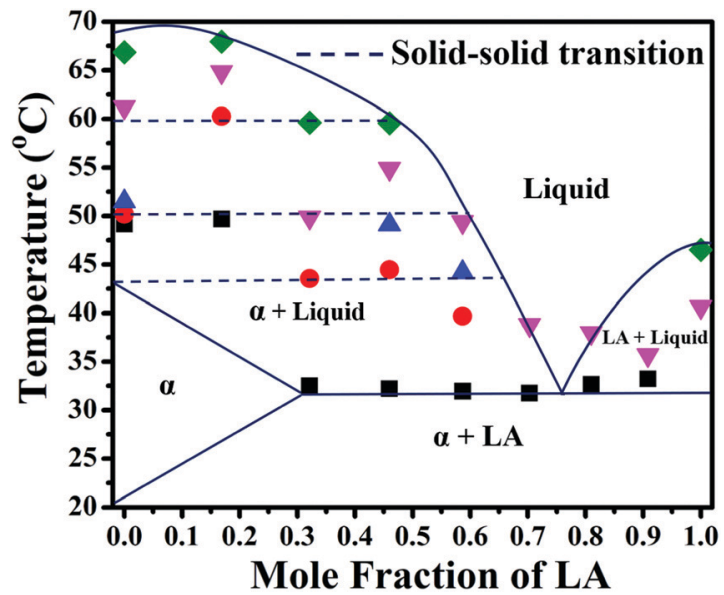

Fig. 10 Phase diagram of the electrospun mat from the second DSC thermal transitions. Dashed lines represent the solid-solid melting transition. The regions between the dashed lines are the two-phase regions where the SA polymorphs and the liquid phases coexist.

and SA showed eutectic behavior with a eutectic temperature and composition being close to, respectively, $30{ }^{\circ} \mathrm{C}$ and $X_{\mathrm{LA}}=0.6$. The eutectic temperature in the nanofibrous PVA was found to be considerably less than that obtained for the bulk mixture $\left(39.5{ }^{\circ} \mathrm{C}\right)$. Based on a simple regular solution model calculation, this difference was attributed to altered interactions between the dissimilar fatty acid molecules in the mat compared with the bulk. In the present study, the eutectic temperature projected from the phase diagram is around $32{ }^{\circ} \mathrm{C}$ at the $X_{\mathrm{LA}}$ composition of around 0.75 . The eutectic composition in the bulk mixture is close to $X_{\mathrm{LA}}=0.85 .{ }^{17}$ We note that with PVA serving as the medium, as in ref. 20, has the highest hydrophilicity; however, in the present work, the PVA-PDMS mixture as the medium has less hydrophilicity and more oleophilicity. ${ }^{16}$ The case of the bulk LA-SA mixture can be considered to have the highest oleophilicity and the least hydrophilicity. The trend in the variation in eutectic composition from 0.6 to 0.75 to 0.85 as the oleophilicity (hydrophilicity) of the medium is enhanced (suppressed) by changing the

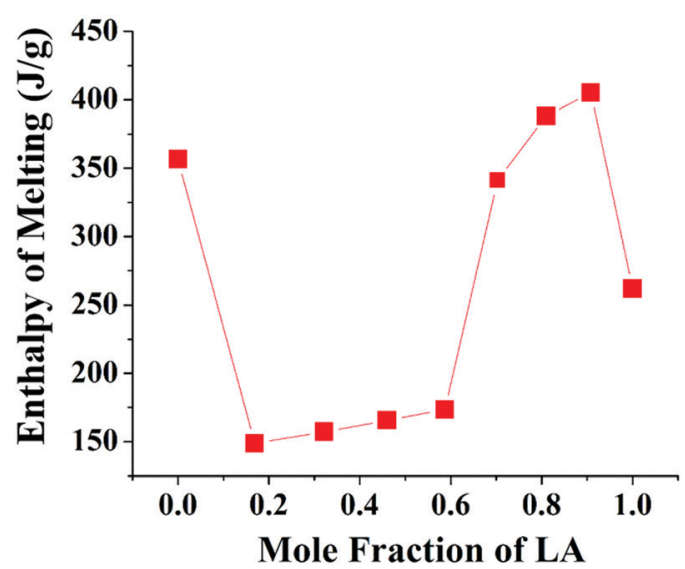

Fig. 11 Heat enthalpy from the second DSC heating scan of the electrospun mat with respect to the energy per unit mass of the dissolved fatty acid component. 
medium from PVA mats to PVA-PDMS mats to bulk suggests that one can engineer the eutectic composition of these mats by changing the hydrophilicity/oleophilicity of the medium.

By virtue of having a high enthalpy of fusion at accessible melting temperatures, the fatty acids are good candidate materials that are useful for thermal energy storage in routine applications and form an important class of "Phase Change Materials". Incorporating these materials in a soft mat can yield sheet-like materials that are potentially useful in the design of thermo-regulating enclosures. The quality of these enclosures will depend upon the transition enthalpy. In Fig. 11, the enthalpy of transition calculated from the area under the thermograms in the second heating scan is presented for different mats. The mats with $X_{\mathrm{LA}}$ close to the eutectic composition show high values of enthalpy. This is in agreement with our prior conclusion drawn from FT-IR and XRD data, that near the eutectic composition, the fatty acid components have a higher interaction and thereby better intermixing.

\section{Conclusion}

In summary, we have successfully fabricated PDMS-PVA nanofibrous mats impregnated with the LA-SA mixtures. The resulting mats show eutectic phase behavior arising from the interactions between the fatty acid components. The PVA-PDMS composite mats can be considered to present these fatty acid molecules in a nano-constrained environment. The eutectic temperature of $32{ }^{\circ} \mathrm{C}$ remains the same as that observed in the PVA mats, but the eutectic composition is found near $75 \mathrm{~mol} \%$ of LA, which is intermediate to the eutectic compositions observed in pure PVA mats and in the bulk. We propose that the hydrophilicity/hydrophobicity of the nanofibrous environment influences the interaction between the fatty acid molecules and thereby the eutectic composition. Pure PVA offers hydrophilic surroundings, whereas in the bulk the LA/SA molecules experience a hydrophobic environment. Incorporating PDMS with the fiber-making PVA, the eutectic composition of the fatty acid mixtures can be altered to various degrees and their phase behaviour can be engineered.

\section{List of abbreviations}

$\begin{array}{ll}M_{\mathrm{w}} & \text { Average molecular weight } \\ \mathrm{CDS} & \text { Cambridge structural database } \\ \mathrm{cm} & \text { Centimeter } \\ \mathrm{cif} & \text { Crystallographic information file } \\ \Phi & \text { Crowding parameter } \\ { }^{\circ} \mathrm{C} & \text { Degree Celsius } \\ { }^{\circ} \mathrm{C} \mathrm{min}{ }^{-1} & \text { Degree Celsius per minute } \\ \text { DSC } & \text { Differential scanning calorimetry } \\ \text { E-spin } & \text { Electrospinning } \\ \text { FE-SEM } & \text { Field-emission scanning electron microscopy } \\ \text { FT-IR } & \text { Fourier transform-infrared } \\ T_{\mathrm{g}} & \text { Glass-transition temperature } \\ \mathrm{kV} & \text { Kilo-volt } \\ \text { LA } & \text { Lauric acid }\end{array}$

$\begin{array}{ll}\mathrm{mg} & \text { milli-gram } \\ \mathrm{mL} \min ^{-1} & \text { milliliter per minute } \\ X_{\mathrm{LA}} & \text { Mole fraction of lauric acid } \\ \text { PVA } & \text { Polyvinyl alcohol } \\ \text { PCM } & \text { Phase change material } \\ \text { Pa s } & \text { Pascal-second } \\ \mathrm{cm}^{-1} & \text { per centimeter } \\ \text { PDMS } & \text { Polydimethylsiloxane } \\ \text { SA } & \text { Stearic acid } \\ \text { wt\% } & \text { Weight percent } \\ \text { XRD } & \text { X-ray diffraction. }\end{array}$

\section{Conflicts of interest}

The authors declare no conflicts of interest.

\section{Acknowledgements}

Authors are grateful to the DST unit on Nanosciences, IIT Kanpur to let us use the XRD, FE-SEM, and DSC instrument facilities. We are thankful to NanoSurf India to help us doing AFM analysis of the mat samples.

\section{References}

1 S. Agarwal, J. H. Wendorff and A. Greiner, Use of electrospinning technique for biomedical applications, Polymer, 2008, 49, 5603-5621.

2 S. S. Ray, S.-S. Chen, C.-W. Li, N. C. Nguyen and H. T. Nguyen, A comprehensive review: electrospinning technique for fabrication and surface modification of membranes for water treatment application, $R S C A d v$., 2016, 6, 85495-85514.

3 A. Greiner and J. H. Wendorff, Electrospinning: A fascinating method for the preparation of ultrathin fibers, Angew. Chem., Int. Ed., 2007, 46, 5670-5703.

4 S. Agarwal, A. Greiner and J. H. Wendorff, Functional materials by electrospinning of polymers, Prog. Polym. Sci., 2013, 38, 963-991.

5 T. Kongkhlang, K. Tashiro, M. Kotaki and S. Chirachanchai, Electrospinning as a New Technique To Control the Crystal Morphology and Molecular Orientation of Polyoxymethylene Nanofibers Electrospinning as a New Technique To Control the Crystal Morphology and Molecular Orientation of Polyoxymethylene, J. Am. Chem. Soc., 2008, 130, 15460-15466.

6 T. Wu, M. Ding, C. Shi, Y. Qiao, P. Wang, R. Qiao, X. Wang and J. Zhong, Resorbable polymer electrospun nanofibers: History, shapes and application for tissue engineering, Chin. Chem. Lett., 2020, 31, 617-625.

7 P. Wang, M. Ding, T. Zhang, T. Wu, R. Qiao, F. Zhang, $\mathrm{X}$. Wang and J. Zhong, Electrospraying Technique and Its Recent Application Advances for Biological Macromolecule Encapsulation of Food Bioactive Substances, Food Rev. Int., 2020, 1-23. 
8 S. K. Sia and G. M. Whitesides, Microfluidic devices fabricated in poly(dimethylsiloxane) for biological studies, Electrophoresis, 2003, 24, 3563-3576.

9 T. Trantidou, Y. Elani, E. Parsons and O. Ces, Hydrophilic surface modification of PDMS for droplet microfluidics using a simple, quick, and robust method via PVA deposition, Microsyst. Nanoeng., 2017, 3, 16091.

10 K. K. Y. Ho, L. M. Lee and A. P. Liu, Mechanically activated artificial cell by using microfluidics, Sci. Rep., 2016, 6, 32912.

11 Y. Y. Song, Y. Liu, H. B. Jiang, S. Y. Li, C. Kaya, T. Stegmaier, Z. W. Han and L. Q. Ren, Bioinspired Fabrication of one dimensional graphene fiber with collection of droplets application, Sci. Rep., 2017, 7, 1-10.

12 T. Lv, Y. Yao, N. Li and T. Chen, Highly Stretchable Supercapacitors Based on Aligned Carbon Nanotube/Molybdenum Disulfide Composites, Angew. Chem., Int. Ed., 2016, 55, 9191-9195.

13 K. F. Lei, K.-F. Lee and M.-Y. Lee, Development of a flexible PDMS capacitive pressure sensor for plantar pressure measurement, Microelectron. Eng., 2012, 99, 1-5.

14 L. Wang, H. Peng, X. Wang, X. Chen, C. Yang, B. Yang and J. Liu, PDMS/MWCNT-based tactile sensor array with coplanar electrodes for crosstalk suppression, Microsyst. Nanoeng., 2016, 2, 16065.

15 K. Phanishwar, S. Perween, N. Saurakhiya and A. Ranjan, Embedded macroporous elastomers by hydrostatic fracturing for flexible strain-sensor applications, J. Appl. Polym. Sci., 2016, 133, 43681.

16 S. Perween, Z. Khan, S. Singh and A. Ranjan, PVA-PDMSStearic acid composite nanofibrous mats with improved mechanical behavior for selective filtering applications, Sci. Rep., 2018, 8, 16038.

17 M. C. Costa, M. Sardo, M. P. Rolemberg, P. Ribeiro-Claro, A. J. A. Meirelles, J. A. P. Coutinho and M. A. Krähenbühl, The solid-liquid phase diagrams of binary mixtures of consecutive, even saturated fatty acids: differing by four carbon atoms, Chem. Phys. Lipids, 2009, 157, 40-50.

18 M. C. Costa, M. P. Rolemberg, L. A. D. Boros, M. A. Krähenbühl, M. G. de Oliveira and A. J. A. Meirelles, Solid-Liquid Equilibrium of Binary Fatty Acid Mixtures, J. Chem. Eng. Data, 2007, 52, 30-36.

19 M. C. Costa, M. Sardo, M. P. Rolemberg, J. A. P. Coutinho, A. J. A. Meirelles, P. Ribeiro-Claro and M. A. Krähenbühl, The solid-liquid phase diagrams of binary mixtures of consecutive, even saturated fatty acids, Chem. Phys. Lipids, 2009, 160, 85-97.

20 R. Gupta, S. Kedia, N. Saurakhiya, A. Sharma and A. Ranjan, Composite nanofibrous sheets of fatty acids and polymers as thermo-regulating enclosures, Sol. Energy Mater. Sol. Cells, 2016, 157, 676-685.

21 H. Liang, B. J. Morgan, G. Xie, M. R. Martinez, E. B. Zhulina, K. Matyjaszewski, S. S. Sheiko and A. V. Dobrynin, Universality of the Entanglement Plateau Modulus of Comb and Bottlebrush Polymer Melts, Macromolecules, 2018, 51, 10028-10039.
22 W. F. M. Daniel, J. Burdyńska, M. Vatankhahvarnoosfaderani, K. Matyjaszewski, J. Paturej, M. Rubinstein, A. V. Dobrynin and S. S. Sheiko, Solventfree, supersoft and superelastic bottlebrush melts and networks, Nat. Mater., 2016, 15, 183-189.

23 T. Subbiah, G. S. Bhat, R. W. Tock, S. Parameswaran and S. S. Ramkumar, Electrospinning of Nanofibers, J. Appl. Polym. Sci., 2005, 96, 557-569.

24 R. Langer, Biomaterials in drug delivery and tissue engineering: One laboratory's experience, Acc. Chem. Res., 2000, 33, 94-101.

25 L. Tian, M. P. Prabhakaran, X. Ding, D. Kai and S. Ramakrishna, Emulsion electrospun vascular endothelial growth factor encapsulated poly(l-lactic acid-co- $\varepsilon^{-}$ caprolactone) nanofibers for sustained release in cardiac tissue engineering, J. Mater. Sci., 2012, 47, 3272-3281.

26 S. Frokjaer and D. E. Otzen, Protein drug stability: A formulation challenge, Nat. Rev. Drug Discovery, 2005, 4, 298-306.

27 C. Shin, G. G. Chase and D. H. Reneker, The effect of nanofibers on liquid-liquid coalescence filter performance, AIChE J., 2005, 51, 3109-3113.

28 C. Shin, Filtration application from recycled expanded polystyrene, J. Colloid Interface Sci., 2006, 302, 267-271.

$29 \mathrm{H}$. Zhao and H. Chi, Novel Aspects of Nanofibers, IntechOpen, 2018, pp. 87-102.

30 E. Moreno, R. Cordobilla, T. Calvet, M. A. Cuevas-Diarte, G. Gbabode, P. Negrier, D. Mondieig and H. A. J. Oonk, Polymorphism of even saturated carboxylic acids from ndecanoic to n-eicosanoic acid, New J. Chem., 2007, 31, 947.

31 S. Sala, E. Elizondo, E. Moreno, T. Calvet, M. A. CuevasDiarte, N. Ventosa and J. Veciana, Kinetically driven crystallization of a pure polymorphic phase of stearic acid from CO2-expanded solutions, Cryst. Growth Des., 2010, 10, 1226-1232.

32 K. Larsson and E. von Sydow, The Crystal Structure of the BForm of Fatty Acids, Acta Chem. Scand., 1966, 20, 1203-1207.

33 V. Vand, W. M. Morley and T. R. Lomer, The crystal sructure of lauric acid, Acta Crystallogr., 1951, 4, 324-329.

34 F. Kaneko, M. Kobayashi, Y. Kitagawa and Y. Matsuura, Collective Displacement of Acyl Chains on E- C PhaseTransition of Stearic-Acid, J. Phys. Chem., 1992, 96, 7104-7107.

35 K. A. Andrianov, G. L. Slonimskii, A. A. Zhdanov, V. Y. Levin, Y. K. Godovskii and V. A. Moskalenko, Some physical properties of polyorganosiloxanes. I. Linear polyorganosiloxanes, J. Polym. Sci., Part A-1: Polym. Chem., 1972, 10, 1-22.

36 A. Habenschuss, M. Tsige, J. G. Curro, G. S. Grest and S. K. Nath, Structure of poly(dialkylsiloxane) melts: Comparisons of wide-angle X-ray scattering, molecular dynamics simulations, and integral equation theory, Macromolecules, 2007, 40, 7036-7043.

37 P. Calcagnile, G. Cacciatore, C. Demitri, F. Montagna and C. E. Corcione, A feasibility study of processing polydimethylsiloxane-sodium carboxymethylcellulose composites by a low-cost fused deposition modeling 3D printer, Materials, 2018, 11, 1-14. 
38 C. Bai, X. Zhang, J. Dai and J. Wang, Synthesis of UV crosslinkable waterborne siloxane-polyurethane dispersion PDMS-PEDA-PU and the properties of the films, J. Coat. Technol. Res., 2008, 5, 251-257.

39 P. Ferreira, Á. Carvalho, T. R. Correia, B. P. Antunes, I. J. Correia and P. Alves, Functionalization of polydimethylsiloxane membranes to be used in the production of voice prostheses, Sci. Technol. Adv. Mater., 2013, 14, 055006.

40 M. Meléndez-Zamudio, A. Villegas, J. A. González-Calderón, R. Meléndrez, M. Meléndez-Lira and J. Cervantes, Study of a Polydimethylsiloxane (PDMS) Elastomer Generated by $\gamma$ Irradiation: Correlation Between Properties (Thermal and Mechanical) and Structure (Crosslink Density Value), J. Inorg. Organomet. Polym. Mater., 2017, 27, 622-632.

41 R. C. L. Mooney, An X-ray Study of the Structure of Polyvinyl Alcohol, J. Am. Chem. Soc., 1941, 63, 2828-2832.

42 T. R. Lomer, The Crystal and Molecular Structure of Lauric Acid (Form A1), Acta Crystallogr., 1963, 16, 984-988.

43 C. W. Bunn, Crystal Structure of Polyvinyl Acohol, Nature, 1948, 161, 929-930.

44 B. Colvin, Crystal Structure of Poly(vinyl alcohol), Nature, 1974, 248, 756-759.

45 T. Hirata and S. Fujita, Effect of Hydrogen Bonding on the Anisotropy of the Hydrostatic Compressibility of Polymer, Crystals, 1979, 17, 1237-1250.

46 E. von Sydow, On the Structure of Crystal Form A of Lauric Acid, Acta Chem. Scand., 1956, 10, 1-8.

47 M. Goto and E. Asada, The Crystal Structure of the A-super Form of Lauric Acid, Bull. Chem. Soc. Jpn., 1978, 51, 70-74.

48 N. Garti, E. Wellner and S. Sarig, Stearic acid polymorphs in correlation with crystallization conditions and solvents, Krist. Tech., 1980, 15, 1303-1310.

49 E. von Sydow, On the structure of the crystal form B of stearic acid, Acta Crystallogr., 1955, 8, 557-560.

50 G. Degermann and E. von Sydow, The thermal Expansion of the Crystalline B- and C-Forms of stearic acid, Acta Chem. Scand., 1959, 13, 984-988.

51 E. von Sydow, Crystallization of Normal Fatty Acids, Acta Chem. Scand., 1955, 9, 1685-1688.

52 M. Goto and E. Asada, The crystal structure of the B-form of stearic acid, Bull. Chem. Soc. Jpn., 1978, 51, 2456-2459.

53 B. D. Mihailova, M. S. Marinov and L. L. Konstantinov, Infrared absorption spectra of rings of $\mathrm{SiO}_{4}$ tetrahedra with imposed boundary conditions, J. Non-Cryst. Solids, 1994, 176, 127-132.

54 M. R. Ahsan, M. A. Uddin and M. G. Mortuza, Infrared study of the effect of $\mathrm{P}_{2} \mathrm{O}_{5}$ in the structure of lead silicate glasses, Indian J. Pure Appl. Phys., 2005, 43, 89-99.

55 W. R. Taylor, Application of infrared spectroscopy to studies of silicate glass structure: Examples from the melilite glasses and the systems $\mathrm{Na}_{20}-\mathrm{SiO}_{2}$ and $\mathrm{Na}_{20}-\mathrm{AI}_{203}-\mathrm{SiO}_{2}$, Proc. - Indian Acad. Sci., Plant Sci., 1990, 99, 99-117.
56 D. Sun, B. B. Li and Z. L. Xu, Preparation and characterization of poly(dimethylsiloxane)-polytetrafluoroethylene (PDMS-PTFE) composite membrane for pervaporation of chloroform from aqueous solution, Korean J. Chem. Eng., 2013, 30, 2059-2067.

57 E. F. dos Reis, F. S. Campos, A. P. Lage, R. C. Leite, L. G. Heneine, W. L. Vasconcelos, Z. I. P. Lobato and H. S. Mansur, Synthesis and characterization of poly (vinyl alcohol) hydrogels and hybrids for rMPB70 protein adsorption, Mater. Res., 2006, 9, 185-191.

58 R. M. Dantzig and M. Rappaz, Solidification, CRD Press, Boca Raton, FL, 2009.

59 B. Nie, J. Stutzman and A. Xie, A vibrational spectral maker for probing the hydrogen-bonding status of protonated Asp and Glu residues, Biophys. J., 2005, 88, 2833-2847.

60 A. Barth, The infrared absorption of amino acid side chains, Prog. Biophys. Mol. Biol., 2000, 74, 141-173.

61 D. L. Pavia, G. M. Lampan and G. S. Kriz, Introduction to Spectroscopy, 3rd edn, 2001.

62 E. Von Sydow, The Infrared Absorption of the Different Crystal Forms of Some Normal Fatty Acids, Acta Chem. Scand., 1955, 9, 1119-1126.

63 Q. Gu, C. Trindle and J. L. Knee, Communication: Frequency shifts of an intramolecular hydrogen bond as a measure of intermolecular hydrogen bond strengths, J. Chem. Phys., 2012, 137, 091101.

64 M. E. L. Gorman, The Evidence from Infrared Spectroscopy for Hydrogen Bonding, J. Chem. Educ., 1957, 34, 304-306.

65 H. Wu, J. V. Volponi, A. E. Oliver, A. N. Parikh, B. A. Simmons and S. Singh, In vivo lipidomics using single-cell Raman spectroscopy, Proc. Natl. Acad. Sci. U. S. A., 2011, 108, 3809-3814.

66 J. L. Lippert and W. L. Peticolas, Laser Raman Investigation of the Effect of Cholesterol on Conformational Changes in Dipalmitoyl Lecithin Multilayers, Proc. Natl. Acad. Sci. U. S. A., 1971, 68, 1572-1576.

67 K. Sato and R. Boistelle, Stability and occurrence of polymorphic modifications of the stearic acid in polar and nonpolar solutions, J. Cryst. Growth, 1984, 66, 441-450.

68 K. Sato, K. Suzuki, M. Okada and N. Garti, Solvent effects on kinetics of solution-mediated transition of stearic acid polymorphs, J. Cryst. Growth, 1985, 72, 699-704.

69 K. Sato, Physical and Molecular Properties of Lipid Polymorphs-A Review, Food Struct., 1987, 6, 7.

70 R. A. Mitran, D. Berger, C. Munteanu and C. Matei, Evaluation of Different Mesoporous Silica Supports for Energy Storage in Shape-Stabilized Phase Change Materials with Dual Thermal Responses, J. Phys. Chem. C, 2015, 119, 15177-15184.

71 W. S. Singleton, T. L. Ward and F. G. Dollear, Physical properties of fatty acids. I. Some dilatometric and thermal properties of stearic acid in two polymorphic forms, J. Am. Oil Chem. Soc., 1950, 27, 143-146. 